\title{
Circadian rhythms of serum atrial natriuretic peptide
}

\author{
Satoru Takeuchi $\cdot$ Hiroshi Nawashiro
}

Received: 9 January 2011 / Accepted: 13 January 2011 /Published online: 1 February 2011

(C) Springer-Verlag 2011

We read with great interest the article entitled "Hyponatremia is predictable in patients with aneurysmal subarachnoid hemorrhage-clinical significance of serum atrial natriuretic peptide" by Nakagawa et al. [1] in the December 2010 issue of the journal.

The authors investigated relationships among hyponatremia, serum atrial natriuretic peptide (ANP) elevation, and the onset of delayed ischemic neurological deficit after subarachnoid hemorrhage (SAH).

The authors' results showed that serum ANP levels at days 0 and 3 after SAH were significantly higher in the hyponatremic patients, whereas the serum sodium level significantly decreased 7 days after SAH. Therefore, the authors suggested that ANP participates in the initiation of hyponatremia and another factor is responsible for cerebral salt-wasting syndrome. We agree with the authors' opinion. We wish to provide further comment on this issue. Sothern et al. [2] reported that, in healthy humans, ANP had significant circadian rhythms with peak concentrations late during sleep being nearly twice the concentrations in the afternoon and evening. We think that if ANP levels at day 0 were analyzed, researchers should attend to the time when blood samples were collected. Differences in ANP levels at day 0 between studies may be attributed to ignorance of these circadian rhythms. Furthermore, we think that researchers should investigate whether circadian rhythms of ANP can be disrupted in SAH patients prior to analysis of ANP at day 0 after SAH.

Conflicts of interest None

\section{References}

1. Nakagawa I, Kurokawa S, Nakase H (2010) Hyponatremia is predictable in patients with aneurysmal subarachnoid hemorrhage - clinical significance of serum atrial natriuretic peptide. Acta Neurochir 152(12):2147-2152

2. Sothern RB, Vesely DL, Kanabrocki EL, Bremner FW, Third JL, Boles MA, Nemchausky BM, Olwin JH, Scheving LE (1995) Blood pressure and atrial natriuretic peptides correlate throughout the day. Am Heart J 129(5):907-916
S. Takeuchi $(\bowtie) \cdot H$. Nawashiro Department of Neurosurgery, National Defense Medical College, 3-2 Namiki,

Tokorozawa, Saitama 359-8513, Japan

e-mail: s.takeuchi@room.ocn.ne.jp 